\title{
Simultaneous Scheduling and Control of Multiproduct Continuous Parallel Lines
}

\author{
Antonio Flores-Tlacuahuac* \\ Departamento de Ingeniería y Ciencias Químicas, Universidad Iberoamericana \\ Prolongación Paseo de la Reforma 880, México D.F., 01210, México \\ Ignacio E. Grossmann \\ Department of Chemical Engineering, Carnegie-Mellon University \\ 5000 Forbes Av., Pittsburgh 15213, PA
}

January 4, 2010

*Author to whom correspondence should be addressed. E-mail: antonio.flores@uia.mx, phone/fax: +52(55)59504074, http://200.13.98.241/ antonio 


\begin{abstract}
In this work we propose a simultaneous scheduling and control optimization formulation to address both optimal steady-state production and dynamic product transitions in multiproduct parallel continuous stirred tank reactors. The simultaneous scheduling and control problem for multiproduct parallel continuous reactors is cast as a Mixed-Integer Dynamic Optimization (MIDO) problem. The reactor dynamic behavior is described by a set of nonlinear ordinary differential equations that are combined with the set of mixed-integer algebraic equations representing the optimal scheduling production model. We claim that the proposed simultaneous scheduling and control approach avoids suboptimal solutions, that are obtained when both problems are solved in a decoupled way. Hence, the proposed optimization strategy can yield improved optimal solutions. The simultaneous approach for addressing the solution of dynamic optimization problems, based on orthogonal collocation on finite elements, is used to transform the set of ordinary differential equations into a Mixed-Integer Nonlinear Programming (MINLP) problem. The proposed simultaneous scheduling and control formulation is tested using three multiproduct continuous stirred tank reactors featuring different nonlinear behavior characteristics.
\end{abstract}




\section{Introduction}

In the context of a highly competitive global market economy, it becomes important to find ways of improving process profit trough improved process operation procedures. In this work, we address a relevant class of industrial problems that involve optimal scheduling and control decisions. Normally, scheduling problems are concerned with the optimal assignments to equipment production sequences, production times for each manufactured product and inventory levels, so that profit or completion times are optimized. On the other hand, control is concerned with determining dynamic transition profiles among the products to be manufactured featuring, for instance, minimum transition time or any other relevant objective function. Commonly, scheduling and control (SC) problems have been approached using a sequential approach: (1) First, the pure scheduling problem is solved using specified transition times and units but without considering the underlying process dynamics of the analyzed system, and (2) having fixed the production sequence, optimal dynamic transition calculations can be carried out among the set of products to be manufactured. Of course, one could reverse this approach and carry out first the control and later the scheduling calculations. However, this approach is only feasible if a small number of product transitions have to be considered. One of the advantages of decoupling scheduling and control problems, lies in the relatively simplicity of solving both problems independently, but the main disadvantage is that generally leads to suboptimal solutions.

To increase production rate, several parallel production facilities can be used. The idea is to distribute the raw material among several equipment in order to increase the throughput. In previous work, we have addressed the simultaneous solution of scheduling and control problems of reaction systems, carried out in a continuous stirred tank reactor (CSTR) [1]. In that work, we presented a mixed-integer dynamic optimization (MIDO) formulation for simultaneously tackling scheduling and control problems, taking place in a single production line. The aim of the present work, consists in extending the single line SC problem, as reported in [1], to reaction systems featuring several parallel production lines each consisting of a CSTR. Only continuous stirred tanks reactors will be used for the purpose of manufacturing the various products. To model the pure scheduling optimization problem we use the parallel lines scheduling formulation proposed in [2], whereas for addressing the optimal control calculations we use the simultaneous approach 
described in [3]. We again propose to solve both problems simultaneously, rather than sequentially, because of the aforementioned reasons. Because the simultaneous SC problem leads to a MIDO problem, and due to the fact that most reaction systems feature nonlinear behavior, it is important to use robust and efficient MIDO solution procedures. As explained in [1], we transformed the SC MIDO problem into a mixed-integer nonlinear program (MINLP). Because of process nonlinearities, the underlying MINLP problem was solved using the sbb software in GAMS [4]. A recent literature review on the solution of scheduling and control problems can be found elsewhere [1].

The research contributions in this work are: (a) Extension of the SC problem from a single production line to multiple parallel production lines, (b) Simultaneously solving the SC problem to avoid suboptimal solutions, (c) Consider the effect of the nonlinear behavior embedded in the reaction systems for the explicit solution of the MIDO problems, and (d) Use a SC MIDO solution decomposition procedure that allows us to compute local optimal solutions with a relatively modest CPU time. This last point is relevant because, if proper MINLP initialization strategies are not used, finding even a feasible solution can be very difficult to achieve. Moreover, no other research work has reported scheduling and control problems in parallel production lines in the open research literature.

\section{Problem definition}

Given are a number of products that are to be manufactured in a fixed number of independent parallel production lines, each one consisting of a single continuous multiproduct CSTR. Lower bounds for constant product demands rates of the products are given, as well as the price of each product and the inventory and raw materials costs. The problem then consists in the simultaneous determination of a cyclic schedule (i.e. production wheel) and the control profile, for the selected transitions for each parallel production line. Steady-state operating conditions for manufacturing each product are also computed. In each production line the major decisions involve selecting the sequence (i.e. cyclic time and the sequence in which the products are to be manufactured) as well as the transition times, production rates, length of processing times, amounts manufactured of each product, and manipulated variables profiles for the transitions such 


\section{Scheduling and Control MIDO Formulation}

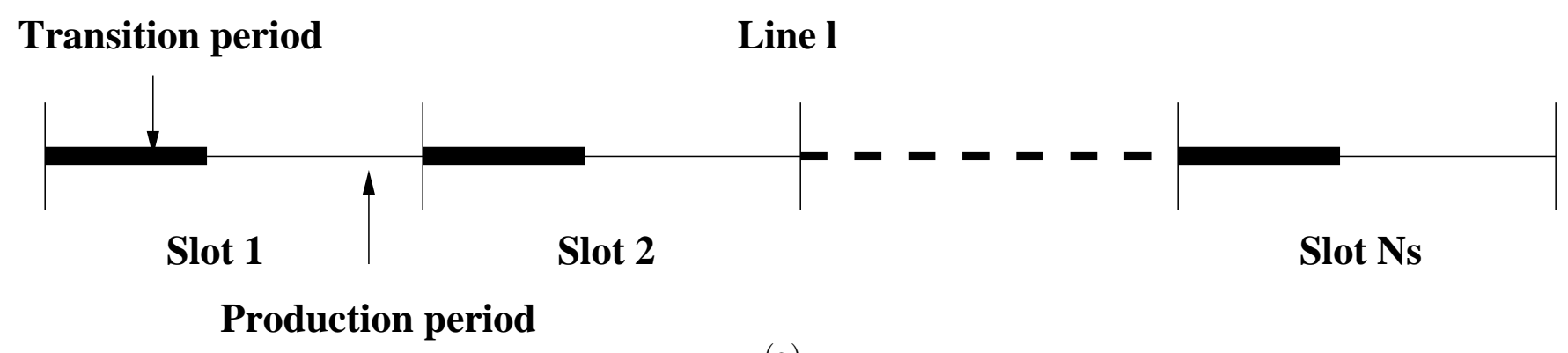

(a)

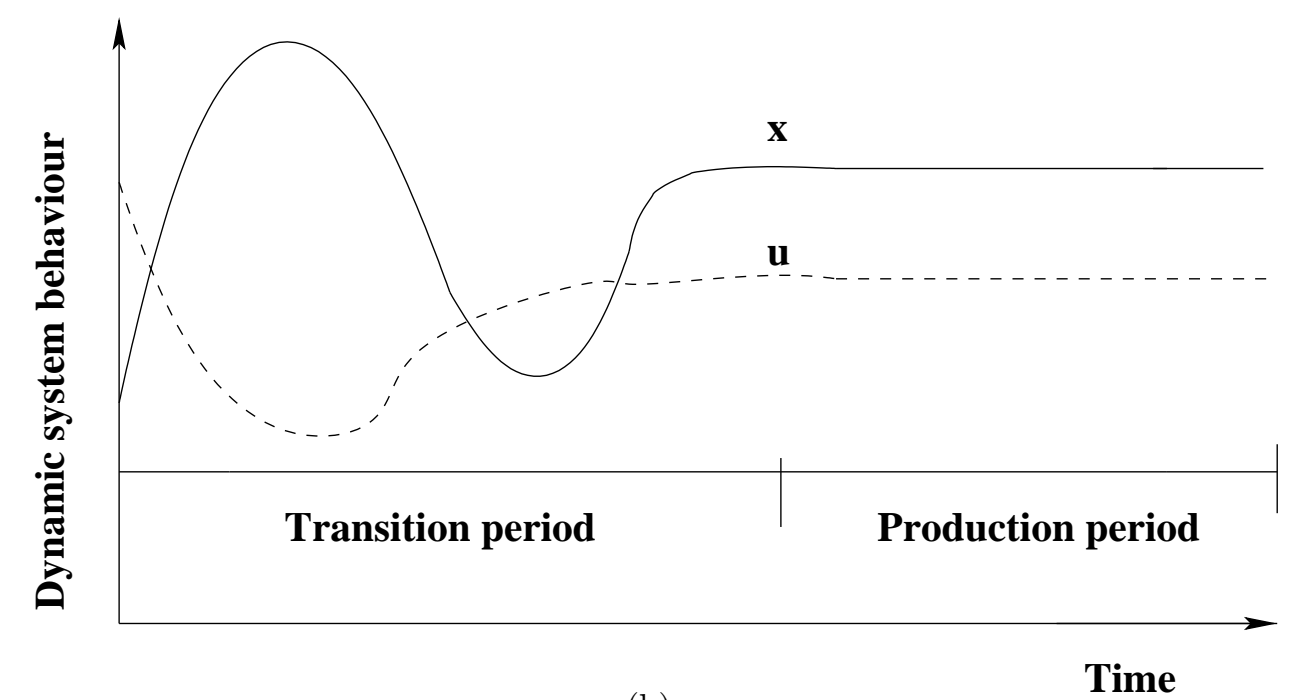

(b)

Figure 1: (a) The process consists of $l$ parallel production lines. At each line $l$ the cyclic time is divided into $N_{s}$ slots. (b) Within each slot a transition period is followed by a steady-state production period, and the system states $x$ and the manipulated variables $u$ remain constant. However during the transition period the manipulated variables change and so does the system states.

In the following simultaneous scheduling and control (SSC) formulation for parallel production plants, we assume that each production line is composed of a single CSTR where desired products are manufactured and that the products follow a production wheel meaning that all the required products are manufactured, in an optimal cyclic sequence (see Sahinidis and Grossmann [2] for the parallel line scheduling formulation). As shown in Figure 1(a) within each production line $l$ the cycle time is divided into a series of time slots. Within each slot two operations take place: (a) the transition period during which dynamic 
transitions between two products take place, and (b) the production period during which a given product is manufactured around steady-state conditions. According to this description, Figure 1(b) depicts a typical dynamic operating response curve within each slot. At the beginning of each slot, the CSTR process conditions are changed (by modifying the manipulated variables $u$ ) until new desired process operating conditions (as represented by the system states $x$ ), leading to the manufacture of a new product, are reached. Afterwards, material of the new given product is manufactured until the demand imposed on such product is met, during this period both the system states $x$ and the manipulated variables $u$ remain constant. In this work we assume that once a production wheel is completed, new identical cycles are executed indefinitely. Note that a given slot does not necessarily incur in a transition. The reason is that it is possible to assign a product to multiple adjacent slots during which a transition does not take place. As an example, the sequence $A B$ with 4 slots may be represented as $A-A-A-B$. It is clear that slots 1 and 2 do not incur in a transition. There are only transitions in slot 3 (from $A$ to $B$ ) and in slot 4 ( $B$ to $A$ due to cyclic schedule). Also, for the particular case when only one product is assigned to a given line, then it means that this line is operating continuously on that line, and hence there is no cyclic schedule on that line. The variable that defines the cycle time in that line becomes undetermined, but causes no numerical difficulties as long as it is bounded.

In order to clarify the simultaneous SC MIDO parallel plants problem formulation, it has been divided into two parts. The first one deals with the scheduling part and the second one with the dynamic optimization part.

\section{Scheduling}

\section{Objective Function}

$$
\begin{aligned}
\min \phi= & \sum_{i} \sum_{k} \sum_{l} c_{i l}^{p} \frac{t_{i k l}^{\prime}}{T c_{l}}+\sum_{i} \sum_{j} \sum_{k} \sum_{l} c_{i j l}^{t} \frac{Z_{i j k l}}{T c_{l}}+\sum_{i} \sum_{k} \sum_{l} c_{i l}^{i} W_{i k l} \\
& +\omega \sum_{i} \Omega_{i}+\alpha_{x} \int_{0}^{t_{f}}\left(\mathbf{x}-\mathbf{x}^{s}\right)^{2} d t+\alpha_{u} \int_{0}^{t_{f}}\left(\mathbf{u}-\mathbf{u}^{s}\right)^{2} d t
\end{aligned}
$$

In the objective function $c_{i l}^{p}$ stands for the production cost of product $i$ in line $l, c_{i j l}^{t}$ denotes the 
transition cost from product $i$ to product $j$ in line $l$, and $c_{i l}^{i}$ is the inventory cost of product $i$ in line $l$ and are given as follows:

$$
\begin{aligned}
c_{i l}^{p} & =c_{i}^{\text {prod }} r_{i l} \\
c_{i j l}^{t} & =-0.9 c_{i l}^{p} \tau_{i j l} \\
c_{i l}^{i} & =c_{\text {saf }}^{i}+0.5\left(1-\frac{d_{i}}{r_{i l}}\right) c_{\text {hold }}^{i}
\end{aligned}
$$

where $c_{i}^{\text {prod }}$ is the production cost of product $i, r_{i l}$ is the production rate of product $i$ in line $l, c_{s a f}^{i}$ is the inventory safety cost and $c_{\text {hold }}^{i}$ is the inventory holding cost. The aim of the integral terms in the objective function is to get a smooth system response, and to penalize for excessive use of those variables associated to the manipulated variables. This means that transitions between different products should be done using the least amount of control action. We have found that including a term related to the dynamic behavior of the states and manipulated variables leads to smooth and fast control actions instead of specifying this desired behavior as constraints. $\alpha_{x}$ and $\alpha_{u}$ are weight coefficients for the states and manipulated variables, respectively. A detailed description of the meaning of each symbol used in this work can be found in the nomenclature section.

\section{(1) Only one product should be manufactured in each slot}

$$
\sum_{i} Y_{i k l}=1, \quad \forall k, \forall l
$$

Constraint 5 states that at any production line $l$ and any slot $k$ only one product $i$ ought to be manufactured. Hence, when the binary variable $Y_{i k l}=1$, product $i$ will be manufactured at slot $k$ within line $l ; Y_{i k l}$ will be zero otherwise. It is worth mentioning that this constraint does not forbid that the same product $i$ be produced in more than one slot $k$ of the line $l$. For instance for two products $A, B$, four slots and a single line, the following product distributions: $A A A B, A A B B$ and $A B B B$ turn out to be the same. Therefore, dummy slots (i.e. slots in which no product manufacture takes place) can sometimes occur. This behavior is illustrated in several of the case studies addressed in this work. 
(2) Transition from product $j$ to product $i$ at slot $k$ and line $l$

$$
\begin{aligned}
\sum_{i} Z_{i j k l} & =Y_{j k l}, \forall j, \forall k, \forall l \\
\sum_{j} Z_{i j k l} & =Y_{i, k--1, l}, \forall i, \forall k, \forall l
\end{aligned}
$$

Constraint [6 is used to define variable $Z_{i j k l}$ that indicates whether a transition from product $j$ to product $i$ is taking place at slot $k$ in line $l$. Hence, if $Z_{i j k l}=1$, a transition will occur between products $j$ and $i$ at slot $k$ within line $l ; Z_{i j k l}$ will be zero otherwise. Constraint 7 states that only one transition from product $j$ will take place at the beginning of slot $k$ at line $l$, if and only if product $j$ was produced in the previous slot.

(4) Each product should be manufactured at least once.

$$
\sum_{l} \sum_{k} Y_{i k l} \geqslant 1, \quad \forall i
$$

Constraint 8 assures that any product $i$ is assigned at least to one slot and one production line. Therefore, any product $i$ is manufactured satisfying production requirements. Notice that because we enforce the greater or equal inequality, any product $i$ can in fact be assigned to more than one slot $k$ within the same line $l$. Similarly, the production of the same product $i$ could be distributed among different lines $l$, as long as production requirements are met.

(5) Upper and lower bounds for production times

$$
\begin{aligned}
t_{i k l}^{\prime} & \leqslant U_{i l} Y_{i k l}, \quad \forall i, \forall k, \forall l \\
\sum_{i} t_{i k l}^{\prime} & \geqslant L_{i l} Y_{i k l}, \quad \forall i, \forall k, \forall l
\end{aligned}
$$

Constraint 9 states that the production time for any product $i$ at any line $l$ and slot $k$ ought to be less or equal that an upper production time bound denoted by $U_{i l}$. Similarly, constraint 10 states a minimum production time, denoted by $L_{i l}$ when manufacturing product $i$ at line $l$ and slot $k$. 
(6) Production time for each line

$$
T c_{l}=\sum_{i} \sum_{k} t_{i k l}^{\prime}, \quad \forall l
$$

Constraint 11 is used to define a variable $T c_{l}$ to record the total production time at each line $l$ (including transitions).

\section{(7) Amount manufactured of each product}

$$
W_{i k l}=r_{i l}\left[t_{i k l}^{\prime}-\sum_{j \neq i} \tau_{j i l} Z_{j i k l}\right], \forall i, \forall k, \forall l
$$

Constraint 12 defines the variable $W_{i k l}$ used to record the amount of product $i$,in mass units, manufactured at line $l$ and slot $k$. This variable is just the product of the process production rate $r_{i l}$ times the net production time. The net production time, computed inside the term in brackets

in the aforementioned equation, is the difference between the total production time $t_{i k l}^{\prime}$ and the transition time. The transition time between products $i$ and $j$, is computed as the product between the transition time $\tau_{j i l}$ and the binary variable $Z_{j i k l}$ used to record is such transition occurs. In pure scheduling problems, $\tau_{j i l}$ is assumed to remain constant, meaning that the dynamic behavior of the addressed system is neglected.

\section{(8) Production demand}

$$
\sum_{l} \sum_{k} \frac{W_{i k l}}{T c_{l}}+\delta_{i} \geqslant d_{i}, \quad \forall i
$$

Constraint 13 states that the total manufactured amount of product $i$ (in mass/time units), taking into account that the production of product $i$ can be distributed among several lines $l$ and slots $k$, ought to be greater or equal to the requested demand $d_{i}$ of such a product $i$. Notice that in this constraint, $\delta_{i}$ stands for a slack variable used to enforce the inequality. Because it should be as small as possible, it is added to the objective function featuring a large coefficient. For cases when $\delta_{i}$ is non-zero at the solution it means that there is no feasible schedule that can be found for the specified demand. 


\section{Optimal Control}

To address the optimal control part, the simultaneous approach [5] for solving dynamic optimization problems was used. In this approach the dynamic model representing the system behavior is discretized using the method of orthogonal collocation on finite elements [6], [7]. According to this procedure, within a line $l$, a given slot $k$ is divided into a number of finite elements. Within each finite element a given number of internal collocation points is selected as depicted in Figure 2, Using several finite elements is useful to represent dynamic profiles with non-smooth variations. Thereby, the set of ordinary differential equations comprising the system model is approximated at each collocation point leading to a set of nonlinear equations that must be satisfied.

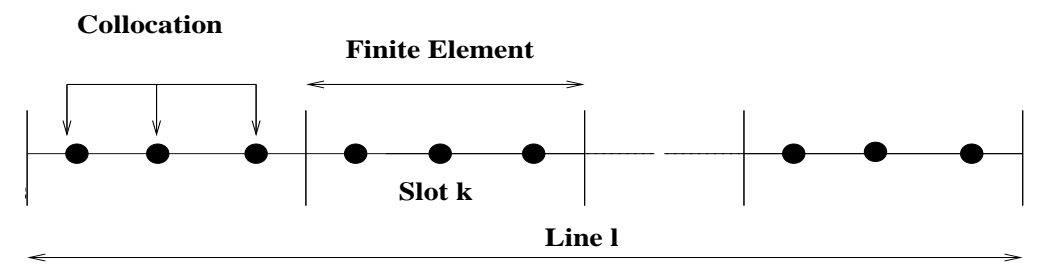

Figure 2: Simultaneous discretization approach for dealing with parallel plants dynamic optimization problems. Each line $l$ is divided into a set of $k$ slots, in turn each slot is divided into $N_{f e}$ finite elements. Within each finite element $f$ a set of $N_{c p}$ collocation points $c$ is selected.

a) Dynamic mathematical model discretization

$$
x_{f c k l}^{n}=x_{o, f k l}^{n}+\theta_{k, l}^{t} h_{f k l} \sum_{m=1}^{N_{c p}} \Omega_{m c} \dot{x}_{f m k l}^{n}, \forall n, f, c, k, l
$$

The constraints given by Equations 14 are used to compute the value of the system states at each one of the discretized points $\left(x_{f c k l}^{n}\right)$ by using the monomial basis representation. $x_{o, f k l}^{n}$ is the $n$-th system state at the beginning of each element, $\Omega_{l c}$ is the collocation matrix and $\dot{x}_{f c k l}^{n}$ is the first order derivative of the $n$-th state. Notice that when working with the first element, $x_{o, 1 k l}^{n}$ represents the specified initial value of the $n$-th state. Also, notice that in the present formulation the length of all finite elements is the same and computed as,

$$
h_{f k l}=\frac{1}{N_{f e}}
$$


b) Continuity constraint between finite elements,

$$
x_{o, f k l}^{n}=x_{o, f-1, k l}^{n}+\theta_{k l}^{t} h_{f-1, k l} \sum_{m=1}^{N_{c p}} \Omega_{m, N_{c p}} \dot{x}_{f-1, m k l}^{n}, \forall n, f \geqslant 2, k, l
$$

In the simultaneous approach for dynamic optimization problems, only the states must be continuous when crossing from one given finite element to the next one; algebraic and manipulated variables are allowed to exhibit discontinuity behavior between adjacent finite elements. That is the reason why continuity constraints are not formulated for algebraic and manipulated variables. We use Equations 16 to force continuous state profiles on all the elements at the beginning of each element $\left(x_{o, f k l}^{n}\right)$ and they are computed in terms of the same monomial basis used before for defining the value of the system states.

c) Model behavior at each collocation point,

$$
\dot{x}_{f c k l}^{n}=f^{n}\left(x_{f c k l}^{1}, \ldots, x_{f c k l}^{n}, u_{f c k l}^{1}, \ldots u_{f c k l}^{m}\right), \forall n, f, c, k, l
$$

Equations 17 are used for computing the value of the first order derivatives of the systems at finite element $f$ of collocation point $c$ in slot $k$ at line $l$. Those equations simply represent the right hand sides of the dynamic model. Because our scheduling and control formulation is system independent, we have used the notation $f^{n}$ to represent the right hand side of the $n$-th ordinary differential equation describing any desired dynamic system. 
d) Initial and final controlled and manipulated variable values at each slot,

$$
\begin{aligned}
x_{i n, 1 l}^{n} & =\sum_{i=1}^{N_{p}} x_{s s, i l}^{n} y_{i, N_{s}, l}, \forall n, l \\
x_{i n, k l}^{n} & =\sum_{i=1}^{N_{p}} x_{s s, i l}^{n} y_{i, k-1, l}, \forall n, k \neq 1, l \\
\bar{x}_{k l}^{n} & =\sum_{i=1}^{N_{p}} x_{s s, i l}^{n} y_{i, k l}, \forall n, k, l \\
u_{i n, 1 l}^{m} & =\sum_{i=1}^{N_{p}} u_{s s, i l}^{m} y_{i, N_{s}, l}, \forall m, l \\
u_{i n, k l}^{m} & =\sum_{i=1}^{N_{p}} u_{s s, i l}^{m} y_{i, k-1, l}, \forall m, k \neq 1, l \\
\bar{u}_{k l}^{m} & =\sum_{i=1}^{N_{p}} u_{s s, i l}^{m} y_{i, k l}, \forall m, k, l \\
u_{1,1, k l}^{m} & =u_{i n, k l}^{m}, \forall m, k, l \\
u_{N_{f e}, N_{c p}, k l}^{m} & =\bar{u}_{i n, k l}^{m}, \forall m, k, l \\
x_{o, 1, k l}^{n} & =x_{i n, k l}^{n}, \forall n, k, l
\end{aligned}
$$

The desired value of each state for each line $l$ at the beginning of the slot $k\left(x_{i n, k l}^{n}\right)$ is computed in Equations 18,19, Equations 20] define the values of the state variables at the end of each slot $k\left(\bar{x}_{k l}^{n}\right)$. It should be stressed that the state values at the beginning and end of each slot $k$ are given by the corresponding steady-state values $\left(x_{s s, i}^{n}\right)$ calculated a priori. $x_{s s, i}^{n}$ simply stands for the steady-state value for the manufacturing of product $i$. They can be easily obtained from open-loop steady-state simulation of the processing system. Similarly, Equations 21 -22 define the values of the manipulated variables at the beginning of each slot $k\left(u_{i n, k l}^{m}\right)$ and at the end of the slot $k\left(\bar{u}_{k l}^{m}\right)$. Equations 23 enforce the system states to take the desired state values at each slot $k$. A similar situation occurs with the values of the manipulated variables. Equations 24 fix the values at the first finite element and first collocation point of each slot $k\left(u_{1,1, k l}^{m}\right)$ as the value that such variable takes at the beginning of the same slot $k$. Equations 25 determine the values of the manipulated variables at the last finite element and last collocation point of slot $k\left(u_{N_{f e}, N_{c p}, k l}^{m}\right)$ as the desired steady-state 
value of the same variable at slot $k\left(\bar{u}_{k l}^{m}\right)$. Finally, Equations 26 determine the values of the system states at the beginning of each slot $\left(x_{o, 1, k l}^{n}\right)$.

e) Lower and upper bounds of the decision variables,

$$
\begin{aligned}
& x_{\text {min }}^{n} \leqslant x_{f c k l}^{n} \leqslant x_{\text {max }}^{n}, \forall n, f, c, k, l \\
& u_{\text {min }}^{m} \leqslant u_{f c k l}^{m} \leqslant u_{\text {max }}^{m}, \forall m, f, c, k, l
\end{aligned}
$$

Equations 27at27b simply constrain the values of both the system states and manipulated variables to lie within acceptable lower and upper bounds.

\subsection{MIDO solution strategy}

In general, the efficient solution of MIDO problems tends to be a rather demanding task. Presently, MIDO solutions for small or medium size mathematical models featuring modest or mild non-linear static and dynamic behavior are relatively easy to compute. However, when it comes to solving large scale models, as those arising from the discretization of distributed parameter systems, that involve highly nonlinear behavior, the MIDO problems turn out to be rather difficult to solve. Moreover, even when such a solution can be computed, it may require large computational times and special initialization and solution procedures. In fact, presently the MIDO solution of complex and large scale problems seems to be feasible only if special optimization decomposition procedures are used [8], [9], [10], [11], [12].

In this work for computing the MIDO solution of the simultaneous scheduling and control problems, the whole MIDO problem was divided into a series of subproblems. In order to do this, we took advantage of the optimization problem structure of the underlying MIDO problems. The optimal solution of each subproblem was then used to provide good initial values of the decision variables of successive versions of the optimization problems. These contain additional modeling details until the complete MIDO problem is finally formulated. The decomposition and MIDO solution strategy involves the following steps: 
1. Solve the parallel lines scheduling problem to obtain an initial scheduling solution using guesses of the transition times and production rates.

2. Compute steady-state processing conditions of the desired products.

3. Solve the dynamic optimization problem to obtain initial optimal transition times and productions rates.

4. Solve the whole MIDO problem for the simultaneous scheduling and control problem.

For the problems addressed in the present work, the aforementioned decomposition and solution strategy allowed us to compute MIDO solutions of the simultaneous scheduling and control problems with acceptable computational effort. It is worth mentioning that if a direct solution strategy is used (meaning the whole solution of the MIDO problem approached in a single step) we have found that sometimes optimal MIDO solutions are impossible to compute, or else the computational times are, in the best case, at least one order of magnitude larger without using a proper initialization strategy. Moreover, it may be hard to find even an initial feasible solution. We think this is partly so, because very good initial values of the decision variables are normally required, for the efficient solution of dynamic optimization problems using the simultaneous approach as described in [3].

\section{Case Studies}

In this section simultaneous SC problems for several case studies, featuring different size and nonlinear behavior, are solved. The problems range in difficulty from a reaction system exhibiting mild nonlinear behavior and involving few products, to a highly complex nonlinear system with a larger

number of products. The problems also differ in the number of processing lines, and the number of postulated slots used for manufacturing the specified products. 


\section{CSTR with a simple irreversible reaction}

Let us assume that in a given chemical plant two production lines are available for carrying out the following reaction system under isothermal and constant volume conditions:

$$
3 R \stackrel{k}{\rightarrow} P,-\mathcal{R}_{R}=k C_{R}^{3}
$$

In each production line a single CSTR can be used, and both reactors have the same dimensions and operating conditions. In principle, any line could manufacture the five desired products denoted as $A, B, C, D, E$. The dynamic composition model is given by,

$$
\frac{d C_{R}}{d t}=\frac{Q}{V}\left(C_{o}-C_{R}\right)+\mathcal{R}_{R}
$$

where $C_{R}$ stands for reactant $R$ composition, $V$ is the reactor volume, $\mathcal{R}_{R}$ is the reaction rate expression, $C_{o}$ stands for feed stream composition and $Q$ is the volumetric flow rate, which is also the control variable used for the optimal dynamic transition between the requested products. The values of the design and kinetic parameters are as follows: $C_{o}=1 \mathrm{~mol} / \mathrm{L}, V=5000 \mathrm{~L}, k=2$ $\mathrm{L}^{2} /\left(\mathrm{mol}^{2}-\mathrm{h}\right)$. In Table 1, the steady-state values of the required reactant conversion percentage are shown. In addition, Table 1 also displays values of the demand rate $\left(D_{i}\right)$, product cost $\left(C_{i}^{p}\right)$ and inventory cost $\left(C_{i}^{s}\right)$. In Figure 3, the bifurcation diagram of the corresponding reaction system is depicted. All the examples in this work require the steady-state values of the manipulated $(u)$ and controlled variables $(x)$ for manufacturing each one of the products, and they are computed as part of the SC solution procedure.

Solving the SC MIDO formulation, we found that in the second production line the optimal production schedule is given by: $D \rightarrow C \rightarrow B \rightarrow D \rightarrow E$, whereas the first production line is completely dedicated to manufacture product $A$. In Table 2, the production results and processing times are shown, whereas in Figure 4 the dynamic optimal transition profiles of both controlled and manipulated variables for the second production line are depicted. It should be remarked that when a product is manufactured in a single line (as is the case in this example for the first production line) 


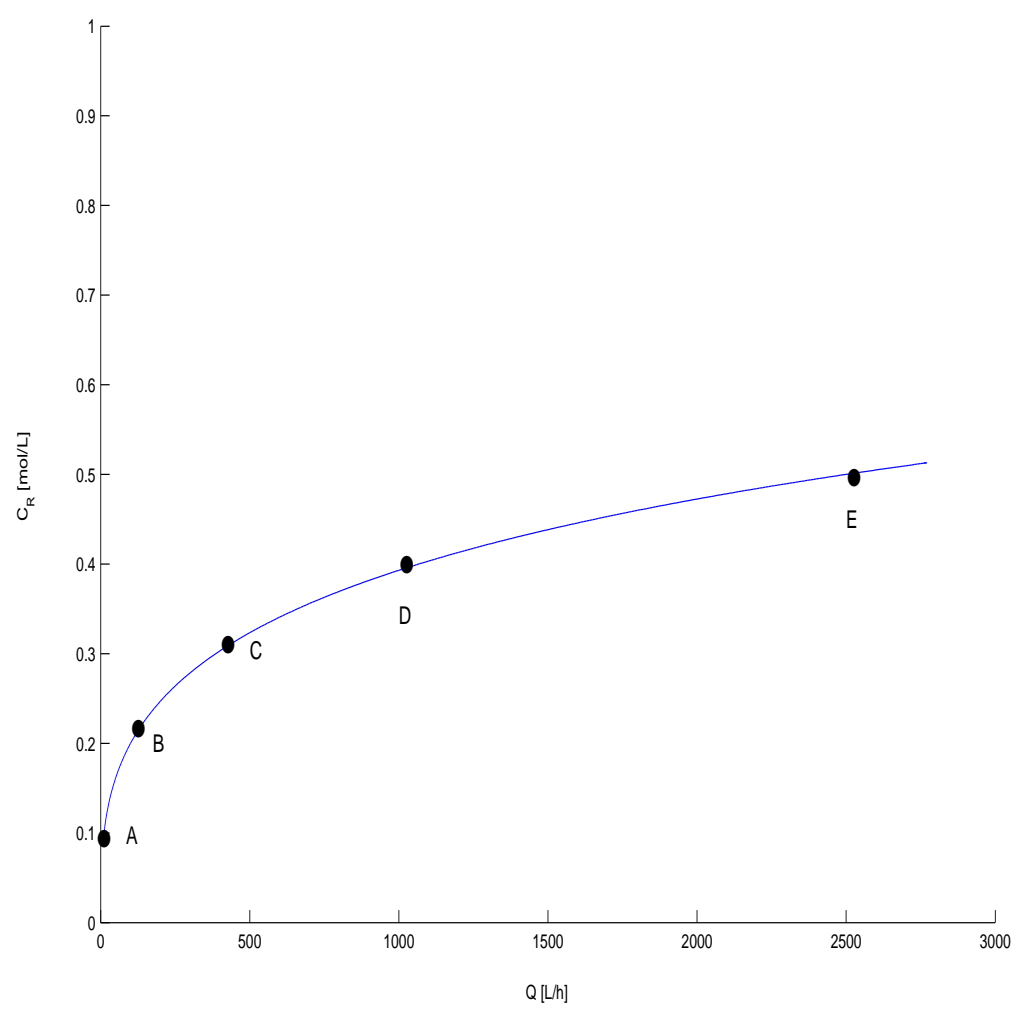

Figure 3: Bifurcation diagram for the first case study: CSTR with a simple irreversible reaction.

the concept of cycle time lacks of meaning. The MIDO problem was solved in $1.29 \mathrm{~h}$ using the 22.7 GAMS optimization version and the SBB MINLP solver with CONOPT as the NLP solver. The MINLP involved 50 binary variables, 3168 continuous variables and 2423 constraints. The optimal objective function value found was $\$ 1.22 \times 10^{5} / \mathrm{h}$ while the NLP relaxation had a value of $\$ 98568.71 / \mathrm{h}$ giving rise to solution gap of $28.58 \%$. Moreover, the number of examined branch and bound nodes was 200. As can be seen in Figure 4, the optimal manipulated variable profile is composed of simple ram-like changes, and therefore smooth responses of the controlled variable are observed.

\begin{tabular}{|ccccc|}
\hline Product & $\begin{array}{c}\text { Conversion } \\
\text { Percentage }\end{array}$ & $\begin{array}{c}\text { Demand } \\
\text { rate }[\mathrm{Kg} / \mathrm{h}]\end{array}$ & $\begin{array}{c}\text { Product } \\
\text { cost }[\$ / \mathrm{kg}]\end{array}$ & $\begin{array}{c}\text { Inventory } \\
\text { cost }[\$ / \mathrm{kg}]\end{array}$ \\
\hline$A$ & 90 & 6 & 200 & 1 \\
$B$ & 80 & 4 & 150 & 1.5 \\
$C$ & 70 & 7 & 130 & 1.8 \\
$D$ & 60 & 6 & 125 & 2 \\
$E$ & 50 & 8 & 120 & 1.7 \\
\hline
\end{tabular}

Table 1: Process data for the first case study. $A, B, C, D$ and $E$ stand for the five products to be manufactured. The cost of the raw material $\left(C^{r}\right)$ is $\$ 10$. 


\begin{tabular}{|c|c|c|c|c|c|c|c|}
\hline Slot & Prod. & $\begin{array}{c}Q \\
{[\mathrm{~L} / \mathrm{h}]}\end{array}$ & $\begin{array}{c}C_{R} \\
{[\mathrm{~mol} / \mathrm{L}]}\end{array}$ & $\begin{array}{l}\text { Process } \\
\text { Time }[\mathrm{h}]\end{array}$ & $\begin{array}{l}\text { Production } \\
\text { rate }[\mathrm{Kg} / \mathrm{h}]\end{array}$ & $\begin{array}{c}\text { Amount } \\
\text { Produced }[\mathrm{kg}]\end{array}$ & $\begin{array}{c}\theta \\
{[\mathrm{h}]}\end{array}$ \\
\hline 1 & $D$ & 1000 & 0.393 & 5.3 & 607 & 165.09 & 2.5 \\
\hline 2 & $C$ & 400 & 0.3032 & 5.7 & 278.8 & 192.6 & 5 \\
\hline 3 & $B$ & 100 & 0.2 & 6.4 & 80 & 110.06 & 3.2 \\
\hline 5 & $E$ & 2500 & 0.5 & 5.2 & 1250 & 220.2 & 3.1 \\
\hline
\end{tabular}

Table 2: Optimal scheduling and control results for the first case study: CSTR with a simple irreversible reaction. Profit $=\$ 1.2246 \times 10^{5} / \mathrm{h}$, Cyclic time at second line $=27.515 \mathrm{~h}$. The results refer to the second production line; the first production line is completely dedicated to manufacture only product $A$. $\theta$ stands for optimal transition time. Note that the fourth slot is used as a transition slot for the transition from product $B$ to product $E$. The transition time at this slot turns out to be equal to $5 \mathrm{~h}$.

There are several points worth mentioning about the optimal solution of this case study. First, as depicted in Figure 3, the system exhibits mild nonlinear steady-state behavior. For each value of the manipulated variable $Q$, there is only a single steady-state value for the whole range of values of the manipulated variable. Moreover, the relationship between the system state and control variable is mildly nonlinear. We think that these facts, in addition to the low dimensionality of the problem, is the main reason why the optimizer finds a SC optimal solution in a relatively short CPU time. These are also the reasons why the shape of the control variables looks to be smooth. To minimize product transition costs, the optimizer decided to manufacture a single product $(A)$ using a complete production line. The production of the rest of the products is distributed in the remaining production line. Moreover, it should be highlighted that actually the fourth slot of the second line is not used for manufacturing product $D$ as reported in the production results displayed in Table 2, The real transition is given by the sequence $D \rightarrow C \rightarrow B \rightarrow E$. The fourth slot is used just as a transition slot in going from product $B$ to product $E$. Even when initially the results of the optimization formulation state that the fourth slot of the second line should be dedicated to manufacturing product $D$, the required demand of product $D$ is completely met at the first slot of the second line as it is clear from the results shown in Table 2. Therefore, the results of the optimization formulation indicate that no production of product $D$ takes place at the aforementioned fourth slot. From constraint 12 we see that this occurs because the processing time $\left(t_{i k l}^{\prime}\right)$ turns out to be equal to the transition time $\left(\tau_{j i l}\right)$ at this slot and, in consequence, the purpose of this slot is just to perform the transition from product $B$ to product $E$. 
Second Line
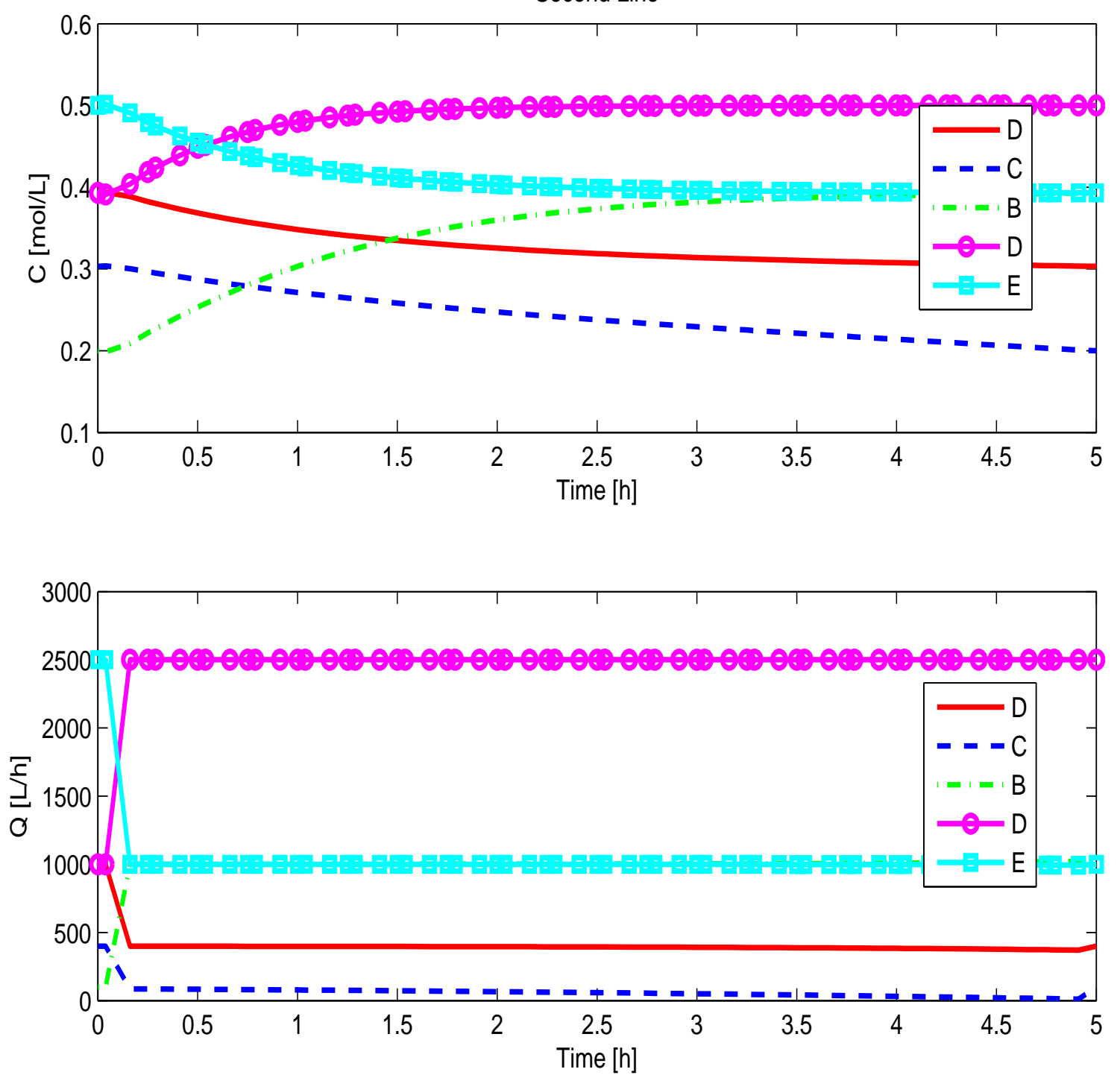

Figure 4: First case study: CSTR with a simple irreversible reaction. Optimal dynamic transition profiles for the controlled and manipulated variables at the second production line for each one of the slots. The optimal production sequence is: $D \rightarrow C \rightarrow B \rightarrow E$

\section{Consecutive reaction system: $X \rightarrow Y \rightarrow Z$}

The second example refers to a nonisothermal continuous stirred tank reactor (CSTR) where the consecutive reactions $X \rightarrow Y \rightarrow Z$ take place [13]. The dynamic behavior of the cooling jacket has also been included. In this problem three production lines and four slots, within each line, are 


\begin{tabular}{|cccccc|}
\hline Product & $\begin{array}{c}\text { Conversion } \\
\text { Percentage }\end{array}$ & $\begin{array}{c}\text { Production } \\
\text { rate }[\mathrm{kg} / \mathrm{h}]\end{array}$ & $\begin{array}{c}\text { Demand } \\
\text { rate }[\mathrm{Kg} / \mathrm{h}]\end{array}$ & $\begin{array}{c}\text { Product } \\
\text { cost }[\$ / \mathrm{kg}]\end{array}$ & $\begin{array}{c}\text { Inventory } \\
\text { cost }[\$ / \mathrm{kg}]\end{array}$ \\
\hline$A$ & 99.84 & 138.7 & 30 & 1 & 1 \\
$B$ & 99.15 & 458.8 & 40 & 3 & 1.5 \\
$C$ & 95.46 & 788.9 & 36 & 2 & 1.8 \\
$D$ & 75.22 & 730 & 44 & 3 & 2 \\
\hline
\end{tabular}

Table 3: Process data for the second case study. $A, B, C$ and $D$ stand for the four products to be manufactured. Three production lines and four slots for each one of the production lines were used.

available. The dimensionless dynamic model is given as follows.

$$
\begin{aligned}
\frac{d x_{1}}{d t} & =q\left(x_{1 f}-x_{1}\right)-x_{1} \kappa_{1}\left(x_{3}\right) \phi \\
\frac{d x_{2}}{d t} & =q\left(x_{2 f}-x_{2}\right)-x_{2} \phi S \kappa_{2}\left(x_{3}\right)+x_{1} \phi \kappa_{1}\left(x_{3}\right) \\
\frac{d x_{3}}{d t} & =q\left(x_{3 f}-x_{3}\right)+\delta\left(x_{4}-x_{3}\right)+\beta \phi\left[x_{1} \kappa_{1}\left(x_{3}\right)+\alpha x_{2} \kappa_{2}\left(x_{3}\right) S\right] \\
\frac{d x_{4}}{d t} & =\delta_{1}\left(q_{c}\left(x_{4 f}-x_{4}\right)+\delta \delta_{2}\left(x_{3}-x_{4}\right)\right) \\
\kappa_{1}\left(x_{3}\right) & =e^{\frac{x_{3}}{1+\frac{x_{3}}{\gamma}}} \\
\kappa_{2}\left(x_{3}\right) & =e^{\frac{\psi x_{3}}{1+\frac{x_{3}}{\gamma}}}
\end{aligned}
$$

where $x_{1}$ is the dimensionless concentration of reactant $X, x_{2}$ is the dimensionless concentration of reactant $Y, x_{3}$ is the dimensionless reactor temperature and $x_{4}$ is the dimensionless cooling jacket temperature. The bifurcation diagram of this reaction system is displayed in Figure 5, Additional dimensionless parameter values are given as follows: $q=1, x_{1 f}=1, x_{2 f}=x_{3 f}=0, x_{4 f}=-1, \beta=$ $8, \phi=0.133, \delta=1, \alpha=1, S=0.01, \psi=1, \delta_{1}=10, \delta_{2}=1, \gamma=1000$. In addition, Table 3 contains information regarding the cost of the products, demand rate, inventory cost, production rate and conversion percentage of each one of the hypothetical products. The variable used as manipulated variable for optimal product transitions is the dimensionless cooling flow rate $q_{c}$.

Solving the SC MIDO formulation we found that the second production line was completely used for manufacturing product $A$, whereas the production of the rest of the products were distributed between the remaining two production lines. In the first production line the optimal production schedule is given by: $D \rightarrow C$, whereas in the third production line the optimal production schedule 


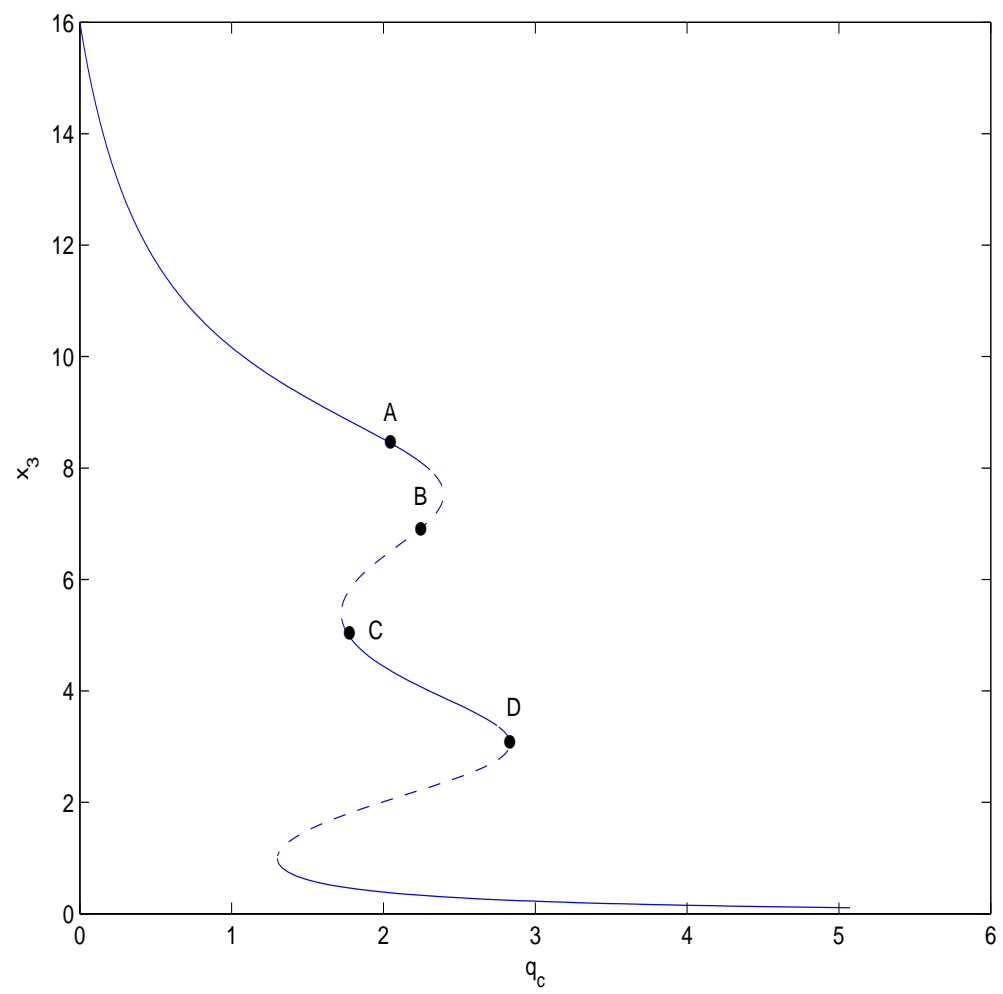

Figure 5: Bifurcation diagram for the second case study: Consecutive reaction system: $X \rightarrow Y \rightarrow Z$. The continuous lines denote open-loop stable steady-states, whereas dashed lines denote open-loop unstable steady-states.

is given by: $A \rightarrow B$. The objective function value is $\$ 874 / \mathrm{h}$. In Figures 6 and 7 the dynamic optimal transition profiles of both controlled and manipulated variables for the first and third productions line are depicted. The MIDO problem was solved in $4.22 \mathrm{~h}$ using the 22.7 GAMS version optimization environment and the SBB MINLP solver. The MINLP involved 48 binary variables, 7996 continuous variables and 7140 constraints. In Table 4, a summary of the results of the local optimal solution are shown.

The results of this case study clearly show the trends of the optimal solution, when a larger number of production lines are used. To minimize transition costs a production line is completely dedicated to manufacturing component $A$. The optimizer also decides to manufacture only two products, at each one of the remaining two production lines. Moreover, it is not a coincidence that the manufactured products at each production line, happen to be close in terms of deviations in both the controlled and manipulated variable as seen in Figure 5. In the limit, with the aim of minimizing transition costs, if we keep adding production lines, we might find a solution where only a single product would 
First Line
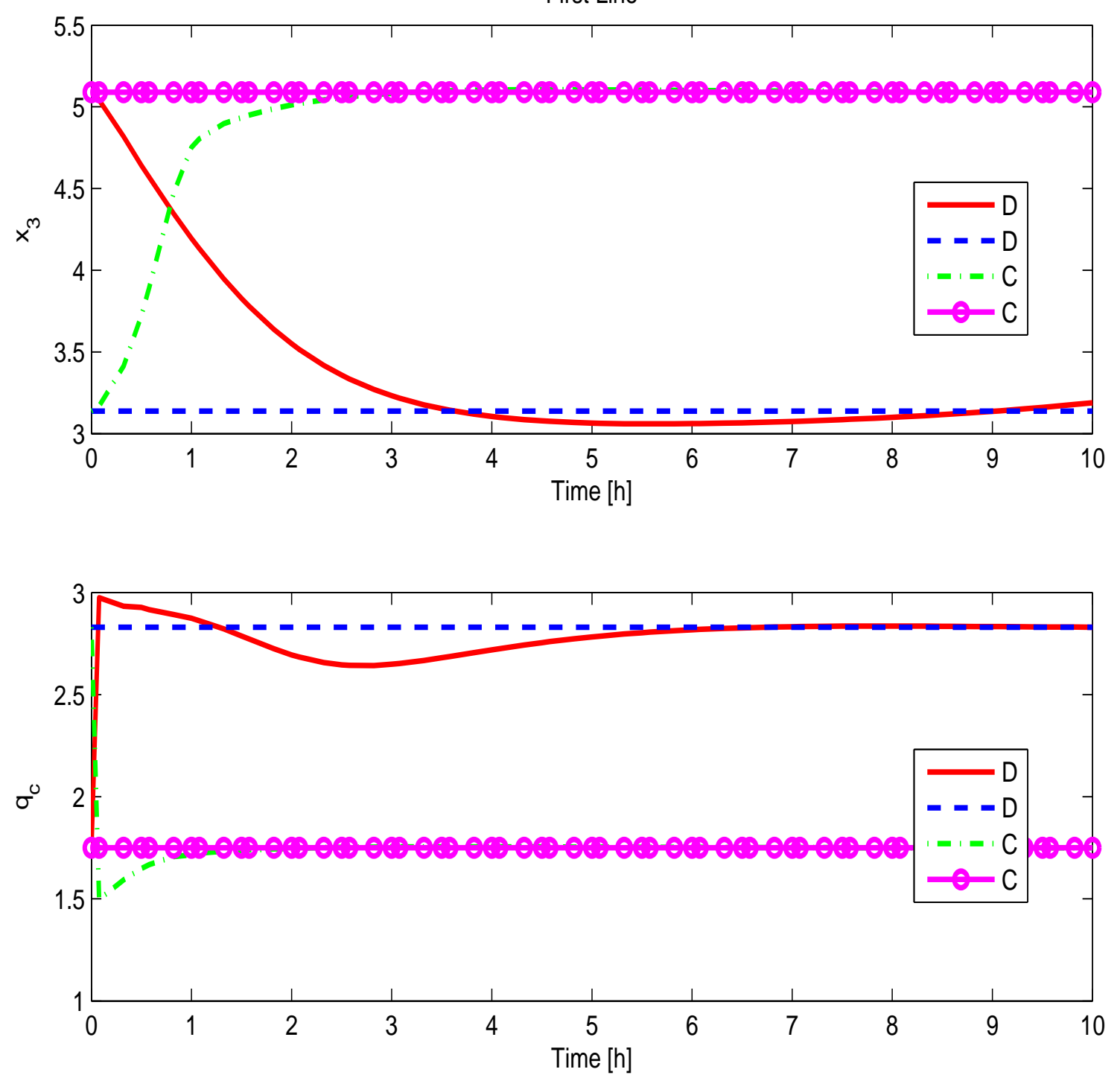

Figure 6: Second case study. Consecutive reaction system optimal dynamic transition profiles for the controlled and manipulated variables at the first production line for each of the slots. The optimal production sequence is: $D \rightarrow C$.

be manufactured at each production line. On the other hand, it is worth to mention that even when the shape of the manipulated variables looks almost symmetrical at both production lines, as seen from Figures 6 and 7 , the system responses are not. This is clear evidence of the nonlinear dynamic behavior of the addressed reaction system. For instance, in Figure 7 , we see that the $A \rightarrow B$ optimal dynamic transition looks different for the same optimal dynamic transition in the opposite direction $B \rightarrow A$. 
Third Line
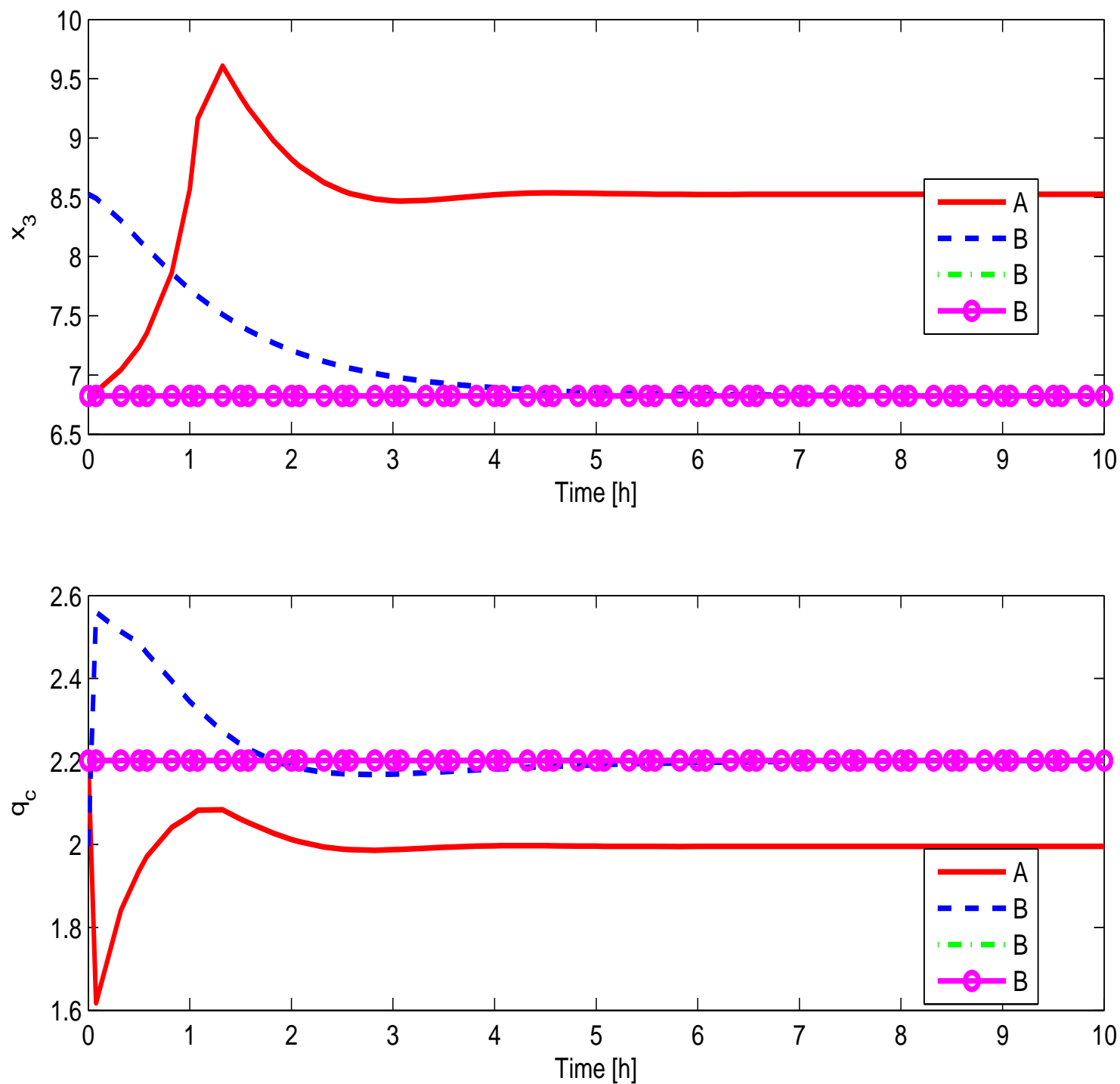

Figure 7: Second case study. Consecutive reaction system optimal dynamic transition profiles for the controlled and manipulated variables at the second production line for each of the slots. The optimal production sequence is: $A \rightarrow B$.

\section{System of 2 series-connected non-isothermal CSTRs}

The next example deals with a system of two cascaded CSTRs where mass and energy is recycled to achieve larger reactant conversion at lower reactor temperatures [14]. In this problem, two production lines and four slots within each line were used. The dimensionless dynamic mathematical model is 


\begin{tabular}{|c|c|c|c|c|c|c|c|c|c|c|c|}
\hline & \multicolumn{5}{|c|}{ Steady-States } & \multicolumn{2}{|c|}{$w[\mathrm{Kg}]$} & \multicolumn{2}{|c|}{$P T[\mathrm{~h}]$} & \multicolumn{2}{|c|}{$\theta[\mathrm{h}]$} \\
\hline Product & $q_{c}$ & $x_{1}$ & $x_{2}$ & $x_{3}$ & $x_{4}$ & $L_{1}$ & $L_{3}$ & $L_{1}$ & $L_{3}$ & $L_{1}$ & $L_{3}$ \\
\hline$A$ & 2 & 0.0016 & 0.1387 & 8.5188 & 2.1729 & & 130.378 & & 10.94 & & 3.3 \\
\hline$B$ & 2.2 & 0.0085 & 0.4588 & 6.8178 & 1.4431 & & 917.6 & & 12 & & 5.1 \\
\hline$C$ & 1.75 & 0.0454 & 0.7889 & 5.0878 & 1.2138 & 805.2 & & 11.021 & & 2.2 & \\
\hline$D$ & 2.83 & 0.2478 & 0.73 & 3.1375 & 0.0803 & 984.238 & & 11.348 & & 8.2 & \\
\hline
\end{tabular}

Table 4: Optimal scheduling and control results for the second case study. Profit $=\$ 874 / \mathrm{h}$. Cyclic times are 22.369 and $22.94 \mathrm{~h}$ for the first and third production lines, respectively. $w$ is the amount manufactured, $P T$ is the processing time. $L_{1}$ and $L_{3}$ stand for the first and third production lines, respectively. $\theta$ stands for optimal transition time.

given as follows:

$$
\begin{aligned}
\frac{d x_{1}}{d t} & =(1-\lambda) x_{2}-x_{1}+D a_{1}\left(1-x_{1}\right) \exp \left(\frac{\theta_{1}}{1+\theta_{1} / \gamma}\right) \\
\frac{d \theta_{1}}{d t} & =(1-\lambda) \theta_{2}-\theta_{1}+D a_{1} B\left(1-x_{1}\right) \exp \left(\frac{\theta_{1}}{1+\theta_{1} / \gamma}\right)-\beta_{1}\left(\theta_{1}-\theta_{c 1}\right) \\
\frac{d x_{2}}{d t} & =x_{1}-x_{2}+D a_{2}\left(1-x_{2}\right) \exp \left(\frac{\theta_{2}}{1+\theta_{2} / \gamma}\right) \\
\frac{d \theta_{2}}{d t} & =\theta_{1}-\theta_{2}+D a_{2} B\left(1-x_{2}\right) \exp \left(\frac{\theta_{2}}{1+\theta_{2} / \gamma}\right)-\beta_{2}\left(\theta_{2}-\theta_{c 2}\right)
\end{aligned}
$$

where $x$ and $\theta$ are the dimensionless concentration and temperature, respectively, the subindex 1 refers to the first reactor, whereas the subindex 2 refers to the second reactor. $D_{a}$ stands for the Damköhler number, $\theta_{c}$ is the dimensionless cooling temperature, $\lambda$ is the recycle ratio, $B$ is the dimensionless heat of reaction, $\gamma$ is the dimensionless activation energy, $\beta$ is the dimensionless heat transfer coefficient. Additional parameter values are given as follows: $\lambda=0.9, \gamma=1000, B=$ $22, \theta_{c 1}=\theta_{c 2}=0, \beta_{1}=2, \beta_{2}=2$. In addition, Table 5 contains information regarding the cost of the products, demand rate, inventory cost, production rate and conversion percentage of each one of the hypothetical products. The bifurcation diagram of this two reactor system is displayed in Figure 8. The variable used as manipulated variable for optimal product transitions is the dimensionless Damköhler number $D_{a}$.

Solving the SC MIDO formulation we found that in the first production line the optimal production sequence is given by: $B_{1} \rightarrow C_{1} \rightarrow D_{1} \rightarrow E_{2}$, whereas in the second production line the optimal production sequence is given by: $A \rightarrow C_{2} \rightarrow B_{2} \rightarrow F$. The objective function value is $\$ 1848 / \mathrm{h}$. 


\begin{tabular}{|cccccc|}
\hline Product & $\begin{array}{c}\text { Conversion } \\
\text { Percentage }\end{array}$ & $\begin{array}{c}\text { Production } \\
\text { rate }[\mathrm{kg} / \mathrm{h}]\end{array}$ & $\begin{array}{c}\text { Demand } \\
\text { rate }[\mathrm{Kg} / \mathrm{h}]\end{array}$ & $\begin{array}{c}\text { Product } \\
\text { cost }[\$ / \mathrm{kg}]\end{array}$ & $\begin{array}{c}\text { Inventory } \\
\text { cost }[\$ / \mathrm{kg}]\end{array}$ \\
\hline$A$ & 51.25 & 450 & 30 & 1 & 1 \\
$B_{1}$ & 60.01 & 600 & 40 & 3 & 1.5 \\
$B_{2}$ & 60.08 & 700 & 45 & 4 & 1.8 \\
$C_{1}$ & 70.08 & 900 & 36 & 2 & 2 \\
$C_{2}$ & 70.04 & 850 & 30 & 4 & 1 \\
$D_{1}$ & 80.02 & 700 & 44 & 3 & 2 \\
$E_{2}$ & 90.05 & 800 & 50 & 5 & 1 \\
$F$ & 98.09 & 750 & 40 & 4 & 1 \\
\hline
\end{tabular}

Table 5: Process data for the third case study. $A, B_{1}, B_{2}, C_{1}, C_{2}, D_{1}, E_{2}$ and $F$ stand for the eight products to be manufactured. Two production lines and four slots for each one of the production lines were used.

\begin{tabular}{|c|c|c|c|c|c|c|c|c|c|c|c|}
\hline & \multicolumn{5}{|c|}{ Steady-States } & \multicolumn{2}{|c|}{$w[\mathrm{Kg}]$} & \multicolumn{2}{|c|}{$P T[\mathrm{~h}]$} & \multicolumn{2}{|c|}{$\theta[\mathrm{h}]$} \\
\hline Product & $x_{1}$ & $\theta_{1}$ & $x_{2}$ & $\theta_{2}^{\dagger}$ & $D_{a}$ & $L_{1}$ & $L_{2}$ & $L_{1}$ & $L_{2}$ & $L_{1}$ & $L_{2}$ \\
\hline$A$ & 0.3629 & 2.3480 & 0.5125 & 1.8795 & 0.047 & & 1537.64 & & 13.42 & & 4.8 \\
\hline$B_{1}$ & 0.0979 & 0.4049 & 0.6001 & 3.8178 & 0.028 & 2083.4 & & 13.48 & & 8.7 & \\
\hline$B_{2}$ & 0.3566 & 2.2594 & 0.6008 & 2.5435 & 0.04837 & & 2306.45 & & 13.3 & & 3.9 \\
\hline$C_{1}$ & 0.0985 & 0.3596 & 0.7008 & 4.5371 & 0.022 & 1875.06 & & 12.09 & & 5.3 & \\
\hline$C_{2}$ & 0.3799 & 2.3774 & 0.7004 & 3.1421 & 0.04664 & & 1537.64 & & 11.81 & & 4.1 \\
\hline$D_{1}$ & 0.1048 & 0.3553 & 0.8002 & 5.2180 & 0.01937 & 2291.74 & & 13.28 & & 4.2 & \\
\hline$E_{2}$ & 0.3533 & 2.0872 & 0.9005 & 4.7090 & 0.0507 & 2604.25 & & 13.26 & & 3.9 & \\
\hline$F$ & 0.9722 & 6.4840 & 0.9809 & 2.2257 & 0.05 & & 2050.18 & & 12.74 & & 6.7 \\
\hline
\end{tabular}

Table 6: Optimal scheduling and control results for the third case study. Profit $=\$ 1848 /$ h. Cyclic times are 52.085 and $51.254 \mathrm{~h}$ for the first and second production lines, respectively. $w$ is the amount manufactured, $P T$ is the processing time. $L_{1}$ and $L_{2}$ stand for the first and second production lines, respectively. $\theta$ stands for optimal transition time. 


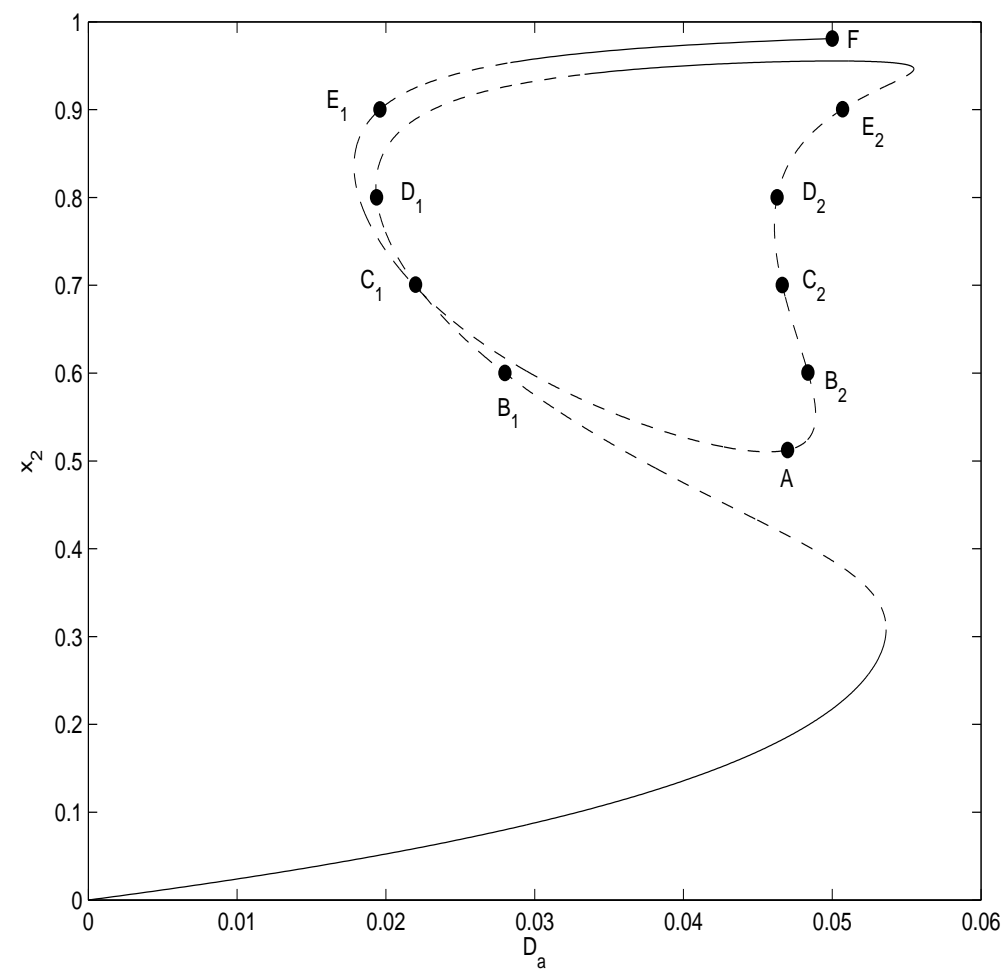

Figure 8: Bifurcartion diagram for the third case study: System of 2 series-connected non-isothermal CSTRs. The continuous lines denote open-loop stable steady-states, whereas dashed lines denote openloop unstable steady-states.

In Figures 9 and 10 the dynamic optimal transition profiles of both controlled and manipulated variables for the first and second production lines are depicted. The MIDO problem was solved in $1.04 \mathrm{~h}$ using the 22.7 GAMS version optimization environment and the SBB MINLP solver. The MINLP involved 64 binary variables, 5995 continuous variables and 5011 constraints. In Table 6, a summary of the results at the local optimal solution are shown.

As noticed from Figure 8, this system features strong nonlinear steady-state behavior. Practically all the required products (except product $F$ ) are manufactured around open-loop unstable operating regions. We should stress that one of the advantages of using the proposed simultaneous SC optimization solution procedure, lies in the fact of its ability to handle dynamic transitions between open-loop unstable steady-states, without introducing a feedback control system whose aim would be first to stabilize the underlying system. More details about this point can be found elsewhere [15]. Moreover, because this problem features a larger number of products, in comparison to the previous case studies, the number of binary variables increases, and so the complexity of solving the 
First Line
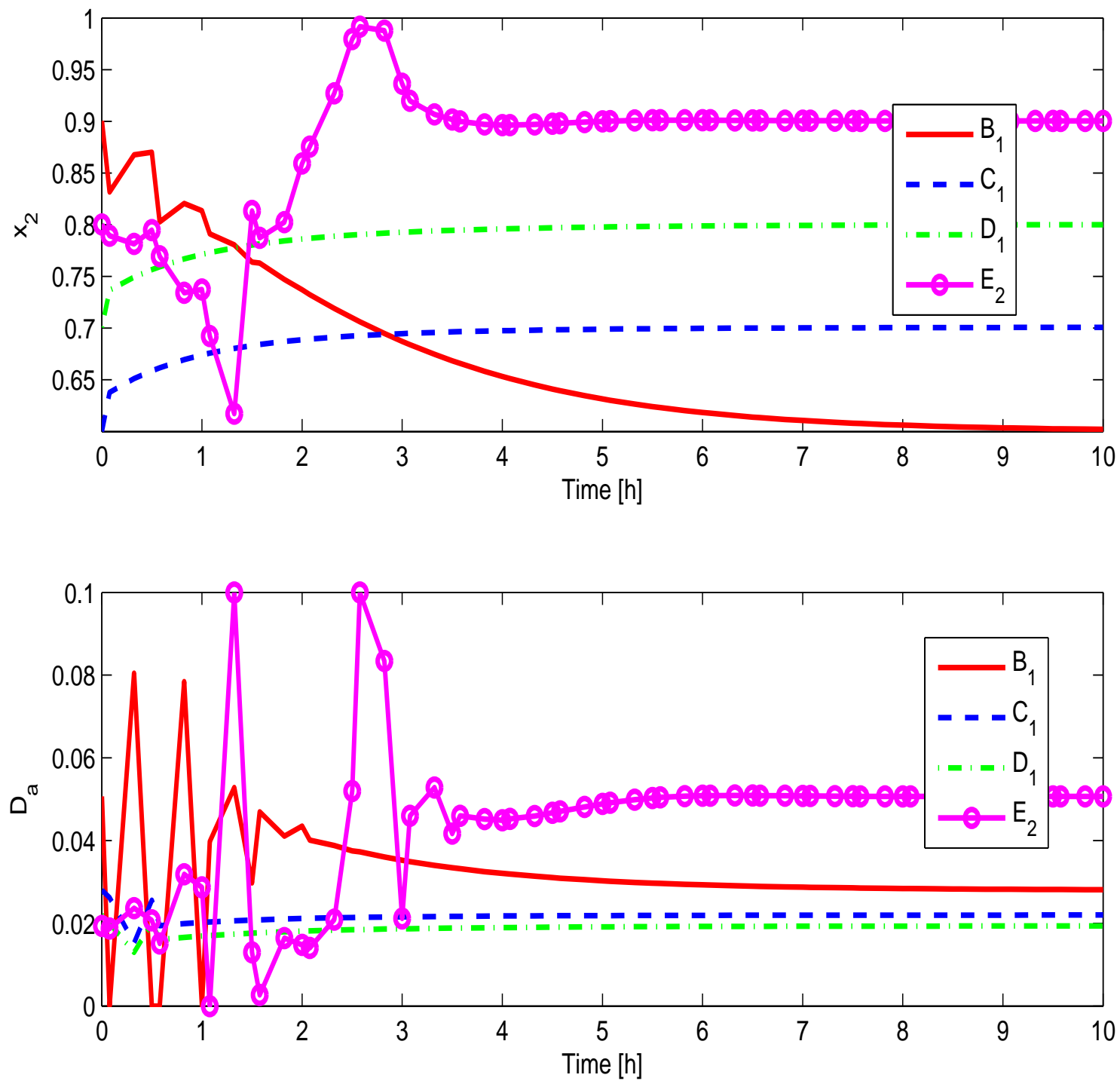

Figure 9: Third case study. Series connected CSTR reaction system optimal dynamic transition profiles for the controlled and manipulated variables at the first production line for each of the slots. The optimal production sequence turns out to be: $B_{1} \rightarrow C_{1} \rightarrow D_{1} \rightarrow E_{2}$.

underlying MIDO problem. However because of the initialization and solution strategy discussed at the beginning of this section, the MIDO problem was solved with a relatively modest computational effort.

It is interesting to see that the simultaneous SC optimal solution involves to manufacture products in an excluding way. This means that four different products are manufactured in a line, whereas the remaining four different products are manufactured in the other production line. The production lines have no common products. Moreover, looking at Figure 8 , we see that the first production 

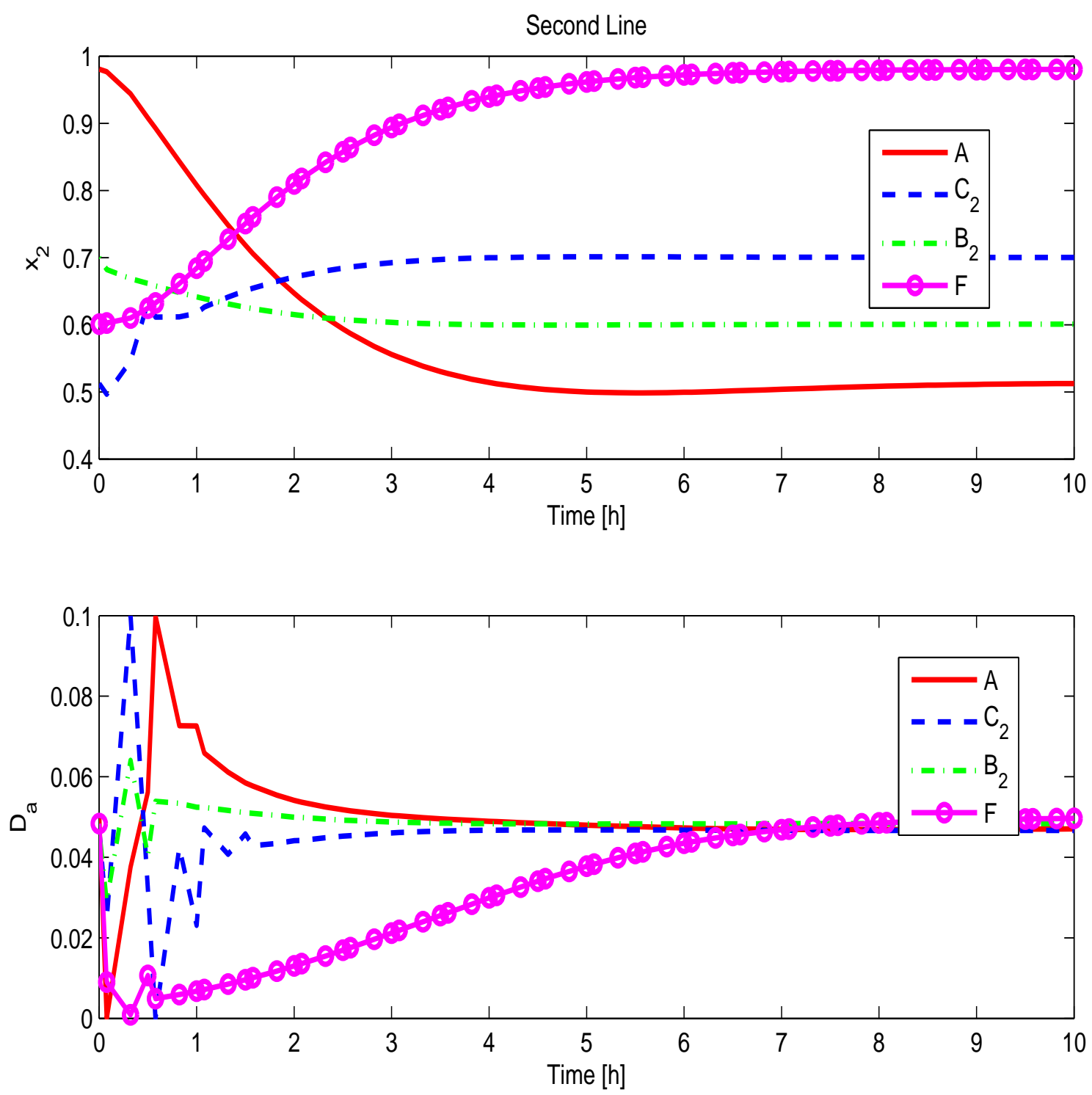

Figure 10: Third case study. Series connected CSTR reaction system optimal dynamic transition profiles for the controlled and manipulated variables at the second production line for each of the slots. The optimal production sequence turns out to be: $A \rightarrow C_{2} \rightarrow B_{2} \rightarrow F$.

line takes care of the products located at the left steady-states branch of this Figure. There is only one product $\left(E_{2}\right)$ that does not belong to this branch, and that is manufactured by this production line. On the other hand, the second production line takes care of those products located at the right steady-states branch. The reason why the simultaneous SC optimal solution involves production lines containing mainly products located on the same steady-state branch is because such product transitions lead to minimum transition costs. This is so because such product transitions feature closer values of the manipulated variables. Therefore, with a relatively modest control effort, we can 
move from a given product to another one located at the same steady-state branch.

Regarding the shape of the optimal dynamic product transition profiles, depicted in Figures 9 and 10, we should highlight that they exhibit relatively smooth dynamic behavior, except in two product transitions (both at the first production line): $D_{1} \rightarrow E_{2}$ and $E_{2} \rightarrow B_{1}$. This result is not surprising because both product transitions involve wide variations in the manipulated variable values. Moreover, these product transitions imply excursions from the left to the right steady-state branches, and viceversa.

\section{Conclusions}

In this work we have extended previously reported work on scheduling and control problems in a single production line [1] to the case of several parallel production lines. We have proposed a simultaneous scheduling and control optimization formulation that upon discretization can be reformulated as an MINLP. We claim that scheduling and control problems should be approached simultaneously rather than in a decoupled way to avoid suboptimal solutions. By using the proposed simultaneous approach to scheduling and control problems, the strong and natural interactions between both problems can be easily taken into account leading to improved optimal solutions. The optimization results indicate the importance of using proper initialization and decomposition procedures, aimed at efficiently solving such problems. In fact, we have found that without proper initialization, it is hard to even find an initial feasible solution. The optimal solution of SC problems of three case studies exhibiting different nonlinear behavior characteristics were presented. These problems were solved with relatively modest computation effort. When attempting to compute global optimal solutions, the computational effort, even for the simpler first case study, turned out to be excessive. This situation suggested that the computation of global optimal solution for the rest of the more complex nonlinear case studies, would demand excessive CPU time and was not further pursued. The development of a global optimal method tailored for simultaneous SC optimization problems remains as a research challenge and we hope to address it in future work. 


\section{Nomenclature}

\section{Indices}

$\begin{array}{ll}\text { Products } & i, p=1, \ldots N_{p} \\ \text { Slots } & k=1, \ldots N_{s} \\ \text { Lines } & l=1, \ldots N_{l} \\ \text { Finite elements } & f=1, \ldots N_{f e} \\ \text { Collocation points } & c, l=1, \ldots N_{c p} \\ \text { System states } & n=1, \ldots N_{x} \\ \text { Manipulated variables } & m=1, \ldots N_{u}\end{array}$




\section{Decision variables}

$y_{i k l} \quad$ Binary variable to denote if product $i$ is assigned to slot $k$ at line $l$

$z_{i p k l}$ Binary variable to denote if product $i$ is followed by product $p$ in slot $k$ at line $l$

$p_{k} \quad$ Processing time at slot $k$

$t_{k}^{e} \quad$ Final time at slot $k$

$t_{k}^{s} \quad$ Start time at slot $k$

$t_{f c k} \quad$ Time value inside each finite element $k$ and for each internal collocation point $c$

$G_{i} \quad$ Production rate

$T c_{l} \quad$ Total production wheel time $[\mathrm{h}]$ at line $l$

$x_{f c k l}^{n} \quad$ N-th system state in finite element $f$ and collocation point $c$ of slot $k$ at line $l$

$u_{f c k l}^{m} \quad$ M-th manipulated variable in finite element $f$ and collocation point $c$ of slot $k$ at line $l$

$W_{i} \quad$ Amount produced of each product $[\mathrm{kg}]$

$\theta_{i k} \quad$ Processing time of product $i$ in slot $k$

$\theta_{k}^{t} \quad$ Transition time at slot $k$

$\Theta_{i} \quad$ Total processing time of product $i$

$x_{o, f k}^{n} \quad n$-th state value at the beginning of the finite element $f$ of slot $k$

$\bar{x}_{k}^{n} \quad$ Desired value of the $n$-th state at the end of slot $k$

$\bar{u}_{k}^{m} \quad$ Desired value of the $m$-th manipulated variable at the end of slot $k$

$x_{i n, k}^{n} \quad n$-th state value at the beginning of slot $k$

$u_{i n, k}^{n} \quad m$-th manipulated variable value at the beginning of slot $k$

$X_{i} \quad$ Conversion 


\section{Parameters}

\begin{tabular}{|c|c|}
\hline$N_{p}$ & Number of products \\
\hline$N_{s}$ & Number of slots \\
\hline$N_{l}$ & Number of lines \\
\hline$N_{f e}$ & Number of finite elements \\
\hline$N_{c p}$ & Number of collocation points \\
\hline$N_{x}$ & Number of system states \\
\hline$N_{u}$ & Number of manipulated variables \\
\hline$D_{i}$ & Demand rate $[\mathrm{kg} / \mathrm{h}]$ \\
\hline$C_{i}^{p}$ & Price of products $[\$ / \mathrm{kg}]$ \\
\hline$C_{i}^{s}$ & Cost of inventory $[\$ / \mathrm{kg}]$ \\
\hline$C^{r}$ & Cost of raw material $[\$]$ \\
\hline$h_{f k}$ & Length of finite element $f$ in slot $k$ \\
\hline$\Omega_{N_{c p}, N_{c p}}$ & Matrix of Radau quadrature weights \\
\hline$\theta^{\max }$ & Upper bound on processing time \\
\hline$t_{i p}^{t}$ & Estimated value of the transition time between product $i$ and $p$ \\
\hline$x_{s s, i}^{n}$ & $n$-th state steady value of product $i$ \\
\hline$u_{s s, i}^{m}$ & $m$-th manipulated variable value of product $i$ \\
\hline$F^{o}$ & Feed stream volumetric flow rate \\
\hline$X_{i}$ & Conversion degree \\
\hline$x_{\min }^{n}, x_{\max }^{n}$ & Minimum and maximum value of the state $x^{n}$ \\
\hline$u_{\min }^{m}, u_{\max }^{m}$ & Minimum and maximum value of the manipulated variable $u^{m}$ \\
\hline & Roots of the Lagrange orthogonal polynomial \\
\hline
\end{tabular}




\section{References}

[1] A. Flores-Tlacuahuac and I.E. Grossmann. Simultaneous Cyclic Scheduling and Control of a Multiproduct CSTR. Ind. Eng. Chem. Res., 45(20):6175-6189, 2006.

[2] N. Sahinidis and I.E. Grossmann. MINLP Model for Cyclic Multiproduct Scheduling on Continuous Parallel Lines. Comput. Chem. Eng., 15(2):85-103, 1991.

[3] L.T. Biegler. An overview of simultaneous strategies for dynamic optimization. Chemical Engineering and Processing, 46(11):1043-1053, 2007.

[4] A. Brooke, D. Kendrick, Meeraus, and R. A. Raman. GAMS: A User's Guide. GAMS Development Corporation, 1998, http://www.gams.com.

[5] L.T. Biegler. Optimization strategies for complex process models. Advances in Chemical Engineering, 18:197-256, 1992.

[6] B. Finlayson. Nonlinear Analysis in Chemical Engineering. McGraw-Hill, New York, 1980.

[7] J. Villadsen and M.Michelsen. Solution of Differential Equations Models by Polynomial Approximation. Prentice-Hall, 1978.

[8] M. Guinard and S.Kim. Lagrangean Decomposition: A model yielding Stronger Lagrangean Bounds. Mathematical Programming, 39:215-228, 1987.

[9] M.L Fisher. The Lagrangian Relaxation Method for Solving Integer Programming Problems. Managament Science, 27(1):2-18, 1981.

[10] A.M. Geoffrion. Lagrangean Relaxation for Integer Programming. Mathematical Programming Study, 2:82-114, 1974.

[11] M.Guignard. Lagrangean relaxation: A short course. J.OR:Special Issue Francoro, 35:3, 1995.

[12] S.Vasantharajan K.Edwards S.A. van den Heever, I.E. Grossmann. A Lagrangean Decomposition Heuristic for the Design and Planning of Offshore Hydrocarbon Field Infrastructure with Complex Economic Objectives. Ind. Eng. Chem. Res., 40:2857-2875, 2001. 
[13] A.E. Gamboa-Torres and A. Flores-Tlacuahuac. Effect of Process Modeling on the Nonlinear Behaviour of a CSTR Reacions $A \rightarrow B \rightarrow C$. Chem. Eng. J., 77:153-164, 2000.

[14] M. Kubicek, H. Hofmann, and V. Hlavacek. Multiplicity and Stability in a Sequence of Two Nonadiabatic Nonisothermal CSTR. Chem. Eng. Sci., 35:987-996, 1980.

[15] A. Flores-Tlacuahuac, L.T. Biegler, and E. Saldívar-Guerra. Optimization of HIPS Open-Loop Unstable Polymerization Reactors. Ind. Eng. Chem. Res., 44:2659-2674, 2005. 